\title{
Imaging the joint in osteoarthritis: a place for ultrasound?
}

\author{
Annamaria Iagnocco, MD, Assistant Professor of Rheumatology*
}

Musculoskeletal Ultrasound in Osteoarthritis, Rheumatology Unit Sapienza University of Rome, Rome, Italy

\section{Keywords:}

musculoskeletal ultrasound

osteoarthritis

synovitis

cartilage lesions

osteophytes

\begin{abstract}
Ultrasound (US) is a valuable tool for imaging musculoskeletal changes in osteoarthritis. It shows early and late findings related to inflammation and structural damage. Sonography is a safe tool, which has recently registered an increasing and widespread use, it being considered as a bedside procedure in the clinical assessment of rheumatic patients. Its applications in osteoarthritis are related to easy accessibility of equipment, low cost, short duration of single examinations and the possibility of performing a multiregional joint evaluation in the same scanning session. Permitting an extensive evaluation of most joint changes present in osteoarthritis, it gives the opportunity to monitor disease progression and perform a follow-up of the response to different local and systemic treatments. US-guided procedures are commonly performed with safety, reliability and optimal patient tolerance. Development in technology and technique with improvement of new research studies will further amplify the diagnostic role of ultrasound in osteoarthritis in the near future.
\end{abstract}

(c) 2009 Elsevier Ltd. All rights reserved.

Musculoskeletal ultrasound (US) represents a novel imaging modality, which has registered an increasing role in rheumatology during the recent years. This is mainly due to tremendous technical advances and progressive, relevant technological developments of US equipment occurring over the past decade [1-5]. Thank to its capacity to represent different anatomic structures in the finest details with consequent possibility to detect an incredible series of changes in minute particulars, sonography seems to represent one of the most promising imaging techniques in rheumatological clinical practice as well as in research imaging [6,7].

\footnotetext{
* Dipartimento di Clinica e Terapia Medica, Cattedra di Reumatologia, Viale del Policlinico 155-00161 Rome, Italy. Tel.: +3906 49974631; Fax: +390649974642.

E-mail address: annamaria.iagnocco@uniroma1.it
} 
However, until recently, the research in the field of US has mainly focussed on the evaluation of inflammatory aspects of rheumatic diseases and on the assessment of tendons and soft tissue involvement $[1,8]$. On the contrary, it has been applied to the study of osteoarthritis (OA) less frequently [9-12]. This issue has been reported in recent reviews that have analysed the published data on the validity of US in assessing inflammatory aspects of rheumatic disorders, underlining the fact that that only a few studies have been undertaken in the field of OA so far [12-14]. Most of these publications refer to the evaluation of synovitis and power Doppler signal, analysing different components of the Outcome Measures in Rheumatology Clinical Trials (OMERACT) filter in rheumatoid arthritis and, in a minority of cases, in OA [12]. However, very recent years have witnessed an increasing interest of research in this field confirming a more widespread use of this tool for imaging different aspects of rheumatic pathology, including OA [15-19].

$\mathrm{OA}$ is a very common rheumatic disorder affecting synovial joints. The main pathological findings are represented by dysregulation of local turnover and changes in repairing processes involving different intra-articular tissues and resulting in the typical global joint involvement. Progressive degeneration with loss of cartilage and hypertrophy of the subchondral bone, joint margin and capsule are the most representative findings of the disease [20]. Synovitis, with a typical episodic course, usually occurs and often contributes to the appearance and worsening of symptoms. Some degrees of cartilage deterioration have also been reported in the presence of synovitis, which has characteristically non-destructive and non-aggressive features, even though it may contribute to symptom aggravation. In most cases, frequent findings are represented by synovial proliferation, joint effusion and bursitis. Usually, OA appears and progressively worsens with the advance of old age, even though it may sometimes occur earlier in life. In those cases, disability and work impairment usually appear prematurely, due to joint use-related pain, swelling, stiffness, deformity and reduced joint motion [20].

OA has been traditionally imaged using conventional radiographs; this has been regarded as the reference technique in OA for a long time $[1,11,17]$. It has, however, clear limitations in imaging and directly visualising hyaline cartilage and other soft tissues, which are frequently involved with disease progression over the years [17,21]. In addition, plain radiographs have very low sensitivity in demonstrating minimal cartilage involvement in early disease. Common radiological findings are represented by joint space narrowing, osteophytes, sclerosis and deformity [20]. However, on the one hand, those features appear sometimes only in late disease and, on the other hand, may be present also in old and asymptomatic people, thus, generating some doubts about their real role and importance as radiographic characteristics of the disease.

Among other imaging modalities, magnetic resonance imaging (MRI) has been demonstrated to be a sensitive and non-invasive technique for evaluating distinct musculoskeletal diseases and has been used as the reference tool in the assessment of criterion validity of US in OA, demonstrating excellent soft-tissue contrasts [12,17,22]. Different studies have demonstrated its accuracy and reliability; however, its high costs and low availability of MRI equipment limit its routine use [11,17]. Arthroscopy is a powerful tool for evaluating most osteoarthritic changes, particularly for direct visualisation of cartilage surface alterations; but its invasiveness limits its use in daily clinical practice [17]. Scintigraphy has shown its predictive value in the assessment of progressive changes in OA; however, it is scarcely available, in a way being invasive and expensive for routine use [20].

\section{Why to use sonography in $O A$ ?}

General indications for using US in OA are reported in Table 1.

In OA, sonography has shown its capability in detecting and evaluating a large number of abnormalities involving hyaline cartilage, synovial fluid and synovial membrane, menisci, joint capsule and bursae as well as in the bony cortex [1,11,21,23-36]. Recent development of high-resolution transducers and more powerful machines has rendered US an emerging and even more widely used tool to image and investigate both early and late changes in $\mathrm{OA}[9,11,17,32,33]$. Its common use as a complementary method for clinical evaluation creates an interesting and useful link between clinical and imaging assessment: it is actually considered a bedside procedure, which can be easily and quickly performed in the meantime and in the same room of the physical examination, thus reducing patient discomfort $[1,4]$. US is a dynamic imaging modality that gives the opportunity to perform a multi-planar 
Table 1

Main indications for using US in OA.

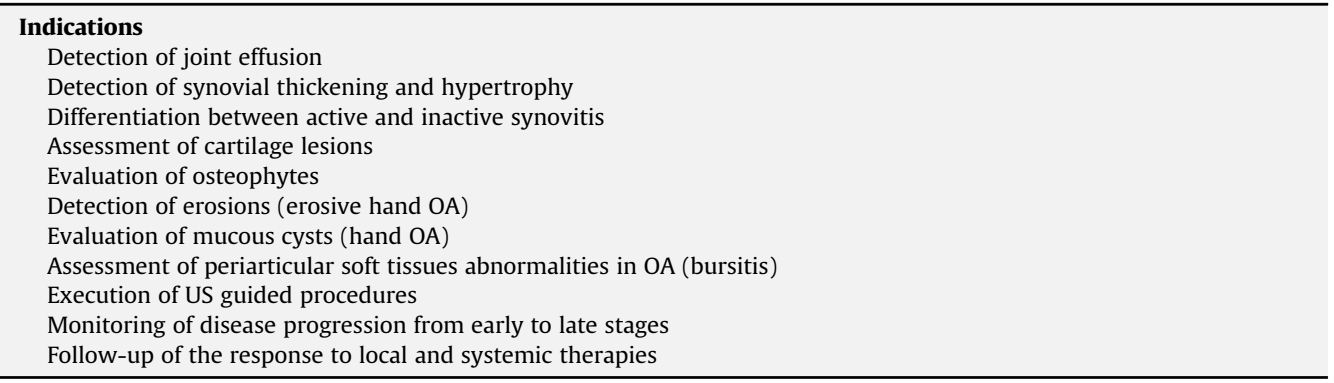

assessment of distinct musculoskeletal areas and most peripheral joints involved in the disease [3]. By using sonography, the clinician is able to directly monitor the progression of the pathology and evaluate the response to therapy by repeating the US examination as many times as necessary with time and during the medical examination $[1,8,11]$. It can be successfully used as a guide for fluid aspirations, injections, biopsies and other diagnostic procedures, improving the reliability and safety of those tools and resulting in an excellent patient tolerance and absence of radiation burden [37-39]. The non-invasiveness and limited cost further improve its routine use in the rheumatological clinical practice during the evaluation of osteoarthritic patients $[3,17]$.

\section{Equipment}

High-quality machines equipped with high-resolution probes represent the necessary requirements for an optimal visualisation of the different musculoskeletal structures, which are commonly involved in OA. Both joint and periarticular soft tissues may be assessed by using different transducers having peculiar technical characteristics. Then, the choice of the most appropriate probe plays a fundamental role for the most satisfactory visualisation of the structures involved in the area under assessment. Most modern US machines are equipped with broadband multi-frequency transducers but the possibility of using even less sophisticated single-frequency probes should be considered as a possible valid alternative, in accordance with the available equipment. In general, approved rules for musculoskeletal US consider high-frequency transducers (more than $12 \mathrm{MHz}$ ) appropriate for the evaluation of small joints and superficial areas while regarding lower-frequency probes (8-12 MHz) as suitable for the assessment of large joints and deep tissues [3,11]. The choice of the more opportune probe size and shape may have a relevant role in some occasions such as while evaluating patients with functional disability and deformities or when assessing wide areas. In fact, small-size transducers, such as hockey stick ones, appear more suitable for the assessment of small joints while large footprint probes are more appropriate for evaluating large joints and wide districts, offering a more panoramic visualisation of the areas of interest [40].

Both gray-scale US and Doppler techniques represent fundamental requirements for the correct evaluation of the osteoarthritic joint. Those procedures represent relevant and sequential steps for the complete assessment of the target areas. After B-mode evaluation by using the most appropriate probe frequency and correct machine setting (image size and depth, gain, focus positioning), colour/power Doppler modalities are applied to assess synovial vascularity, which may be increased in case of active inflammation within the joint and other synovial periarticular structures [3,11,41]. Appropriate grayscale and power Doppler settings are reported in Table 2. Even in OA as well as in inflammatory arthritis, Doppler techniques consent to demonstrate local hyperaemia due to active synovial inflammation [42-45]. A fundamental aspect when using Doppler modalities consists of the application of the optimal setting, which markedly improves the capacities of US in detecting increased flow in inflammatory pathological conditions [41]. In particular, the use of the correct Doppler frequency (high frequencies for superficial tissues and low frequencies for deep structures), the application of the lowest pulse repetition frequency, the adjustment of the appropriate colour gain, the positioning of the 
Table 2

Gray-scale and power Doppler setting.

\begin{tabular}{|c|c|}
\hline Gray-scale setting & Power Doppler setting \\
\hline $\begin{array}{l}\text { Higher probe frequency ( } \geq 14 \mathrm{MHz} \text { ) for superficial tissues and } \\
\text { small joints }\end{array}$ & $\begin{array}{l}\text { Higher Doppler frequency }(7.5-12 \mathrm{MHz}) \\
\text { for superficial tissues }\end{array}$ \\
\hline $\begin{array}{l}\text { Lower frequency ( } \leq 12 \mathrm{MHz} \text { ) for deep structures and large } \\
\text { joints }\end{array}$ & $\begin{array}{l}\text { Lower Doppler frequency }(5-7 \mathrm{MHz}) \\
\text { for deep structures }\end{array}$ \\
\hline Maximal image size (depth) to visualise even minimal details & $\begin{array}{l}\text { Lowest possible pulse repetition } \\
\text { frequency }(0.5-1 \mathrm{KHz})\end{array}$ \\
\hline $\begin{array}{l}\text { Medium image size (depth) to obtain a more panoramic view of } \\
\text { the area of interest }\end{array}$ & $\begin{array}{l}\text { Optimal colour gain (just below the level } \\
\text { causing noise artefacts) }\end{array}$ \\
\hline $\begin{array}{l}\text { Gain adjustment to low-medium levels to obtain optimal } \\
\text { visualization of different tissues, avoiding artefacts }\end{array}$ & $\begin{array}{l}\text { Correct positioning of the foci, at the } \\
\text { level of the area of interest }\end{array}$ \\
\hline $\begin{array}{l}\text { Variable number of foci (usually from } 1 \text { to } 3 \text { ) according to the } \\
\text { extent of the target area }\end{array}$ & $\begin{array}{l}\text { Appropriate modification of the colour } \\
\text { box size according to the extent of the } \\
\text { studied area, including the upper part } \\
\text { of the image to avoid reverberation artefacts }\end{array}$ \\
\hline Correct positioning of the foci, at the level of the area of interest & \\
\hline
\end{tabular}

focus by the area of interest and the correct regulation of the colour box size represent all the fundamental aspects to be considered $[41,45]$.

In general, it is actually possible to assert that past technological advances in US equipment, which have led to recent considerable improvement in software and hardware and the production of highresolution transducers have sensibly amplified the diagnostic abilities of US in rheumatology [11].

\section{Technique}

Guidelines for US in rheumatology have been published in 2001 and represent the reference point in sonographic assessment of the joints in OA [46]. An adequate knowledge of the scanning technique for the various anatomic areas to be examined is mandatory to correctly evaluate the different joints.

A standard scanning protocol, including multi-planar, dynamic and bilateral assessment, should be always followed to perform a complete study of the various anatomic structures included in the examined joint [8].

The use of generous amounts of ultrasonographic gel is necessary for improving the visualisation of the structures included in the target area and reducing occurring artefacts.

Correct patient positioning is fundamental for the best visualisation of different joint tissues. In particular, for the imaging of the hyaline cartilage, joints should be kept in well-defined and standardised positions to enable the ultrasonographic beam to penetrate through the most suitable acoustic windows [46]. A tailored protocol for a complete study of the joints in OA includes the assessment of hyaline cartilage, bony cortex, synovial fluid and synovial membrane for detection of cartilaginous lesions, osteophytes, erosions and synovitis [11]. In target areas where bursitis may appear in OA with the presence of Baker's cysts and bunion bursitis, those particular pathological conditions should be investigated [1]. The documentation of all lesions by two perpendicular planes is mandatory [11].

\section{US of the normal joint}

The normal joint space is imaged by US as an area containing minimal amounts of hypo-anechoic synovial fluid, being limited, on the one hand, by hyperechoic and regular margins that correspond to the bony cortex, and, on the other hand, by a homogeneously echoic band representing the joint capsule $[1,3,11]$. The assessment of synovial fluid is possible by performing multi-planar and dynamic scans and comparing findings with the contralateral side [1-3,5]. This useful scanning protocol, which should always be applied, facilitates a correct evaluation of the joint and avoids errors or misinterpretations due to the fact that minimal amounts of fluid are commonly present in the joints of healthy individuals. Standard reference values for measurements of normal joints have been recently reported and are useful for differentiations in clinical practice [47]. 
By using specific acoustic windows through which the ultrasonographic beam penetrates and keeping the joints in the correct position, articular cartilage may be imaged in most articular sites. Usually, the proper patient position for the assessment of the cartilage consists of keeping the joint either in maximal flexion (hand and knee) or in extension (elbow, wrist, ankle and foot) or in intra-/ extra-rotation (hip and shoulder). Due to its high water content, it typically appears as a homogeneously anechoic band with curvilinear shape $[3,48]$. Its anterior margin is typically sharp, regular and continuous and represents the interface between cartilage and soft tissues. It is thinner than the posterior edge, which is more echoic and thicker and corresponds to the interface between cartilage and bony profile $[1,11]$. Thanks to its typical ultrasonographic characteristics, which depict the cartilage as a well-defined anechoic structure lacking internal echoes, it is possible to measure its thickness that sensibly differs in the various sites, according to the size of the joint where it is measured; it usually varies between 0.1 and $0.5 \mathrm{~mm}$ in the small hand joints to $3 \mathrm{~mm}$ in the knee $[40,48]$. In some deep joints lacking appropriate acoustic windows, hyaline cartilage cannot be imaged by US, except in limited portions that may not be representative of the entire structure. To optimally visualise the cartilage, a correct, perpendicular insonation of the US beam is mandatory [40].

In some joints, menisci can be visualised as homogeneously echoic triangular structures located in the inner part of the joint space, between the bones [11,48].

\section{US of the osteoarthritic joint}

Sonography demonstrates a large set of changes involving the hyaline cartilage from early to late disease (Fig. 1a). Initial findings are represented by blurring of the edges, which become irregular and lose their normal sharpness $[1,11,17]$. Firstly, they involve the superficial cartilaginous margin and correspond to the micro-cleft formation due to tissue deterioration [49]. Later, changes in the echotexture appear, with evidence of loss of homogeneity and transparency [22,49-53]. With disease progression, focal and asymmetric narrowing is usually present; subsequently, diffuse thinning is charted, up to the complete absence of the cartilaginous layer that corresponds to cartilage breakdown and bony denudation [54-57]. In the presence of joint effusion, fluid placing over the superficial margin of the cartilage may create pseudo-thickening; this particular finding should be correctly identified to avoid errors and misinterpretations [1]. All cartilaginous changes need to be assessed by using a correct US scanning technique, based on appropriate patient positioning to concentrate the sonographic beam to penetrate the joint, adequate probe location to obtain perpendicular insonation of the US beam and assessment of the contralateral site to perform complete and deep comparisons [1,3,46].

Osteophytes are imaged by US as cortical protrusions at the joint margin (Fig. 1b) seen in two planes and visualised as either proximal or distal to the joint [18]. They usually have a posterior acoustic shadow [3]. In erosive hand OA, erosions are imaged as a cortical breakage with a step-down contour defect, seen in two planes $[1,15]$. They can be detected with varying degrees of clarity related to the interposition of osteophytes, which may determine narrowing of the acoustic window [1,15].

In osteoarthritic patients with synovitis, joint effusion with synovial thickening and proliferation appears and they can be both detected by gray-scale US (Fig. 1c). In the presence of active inflammation, Doppler modalities are able to demonstrate increased local vascularisation (Fig. 1d) within the synovial tissue $[41-43,45]$. In general, OMERACT definitions for synovial fluid and synovial hypertrophy in rheumatoid arthritis can be applied in OA as well [11,58]. Sonography is able to identify even minimal joint effusion, which can be either anechoic or inhomogeneously hypoechoic, depending on its composition and presence of intra-articular debris and proteinaceous or calcified material $[1,59]$. Synovial hypertrophy, classically defined as a non-displaceable and poorly compressible tissue, is a frequent finding in inflamed osteoarthritic joints.

In patients with knee OA, US scans over the medial aspect of the joint frequently demonstrate protrusion of the medial meniscus that determines displacement of the medial collateral ligaments; this finding seems to be correlated with joint space narrowing [21].

On some occasions, using appropriate scans, joint capsule thickening may be imaged; this particular finding, related to capsule hypertrophy and fibrosis, seems to be a characteristic change in osteoarthritic joints; comparisons with the contralateral side are mandatory to avoid errors and misinterpretations [8]. 

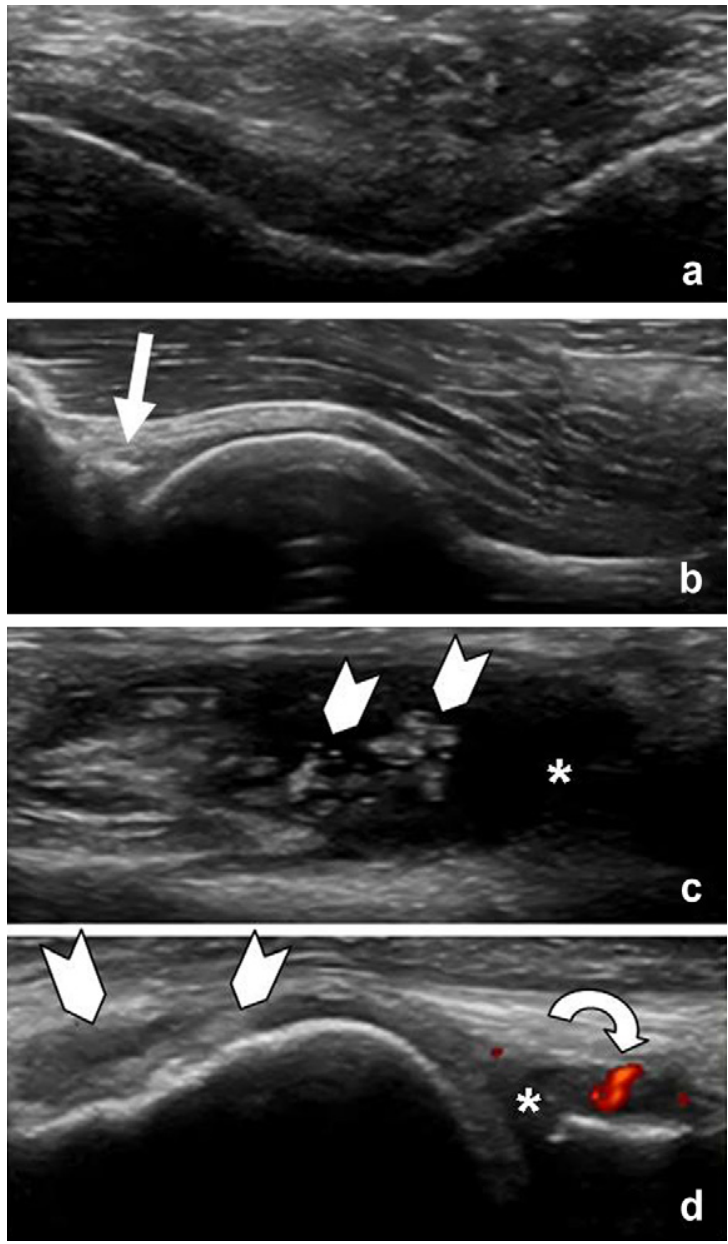

Fig. 1. Sonographic evaluations of osteoarthritic joints. a: $12 \mathrm{MHz}$ US image of the femoral condylar hyaline cartilage. Anterior transverse scan in the suprapatellar area with the joint fully flexed. Note the presence of blurring and irregularities of the superficial and deep interfaces with loss of their normal sharpness; changes in the cartilage homogeneity and transparency; asymmetric cartilage thinning. b: $9 \mathrm{MHz}$ US image of the hip joint. Anterior longitudinal scan. Note the presence of a large osteophyte (arrow) localised over the acetabular side of the joint. c: $12 \mathrm{MHz}$ US images of the lateral para-patellar recess of the knee. Lateral oblique scan. Note the presence of local effusion $\left(^{*}\right)$ and synovial proliferation (arrowheads) which assumes the aspect of vegetations with varying morphology. d: $15 \mathrm{MHz}$ image of the first metatarsophalangeal joint. Anterior longitudinal scan. Power Doppler shows the presence of local active inflammation by the detection of intra-articular power Doppler signal (curved arrow). Note the presence of joint effusion $\left({ }^{*}\right)$ and synovial proliferation (arrowheads).

In the hand, OA mucous cysts may be depicted by sonography, which demonstrates excellent correlations with clinical findings and shows the presence of characteristic hypoechoic formations with well-defined margins, located over the superolateral aspect of the distal interphalangeal joints [1,9,36].

To complete US evaluation of osteoarthritic joints, analysis of periarticular areas, where abnormalities may be occasionally found, is necessary [1,11,48]. Typically, those changes consist of the appearance of bursitis that is imaged by US with the representation of abnormal hypo-anechoic, displaceable and compressible intrabursal material. They usually involve the local bursae of the knee, with appearance of Baker's cysts and anserine bursitis, and the foot with demonstration of bursitis over the medial aspect of the first metatarsophalangeal joint $[1,11]$. 
Table 3

Advantages and disadvantages of US.

\begin{tabular}{ll}
\hline Advantages & Disadvantages \\
\hline $\begin{array}{l}\text { Safety, non-invasiveness, no radiation burden } \\
\text { Low running cost }\end{array}$ & $\begin{array}{l}\text { Limited number and width of acoustic windows } \\
\text { Operator dependency } \\
\text { Absence of contra-indications }\end{array}$ \\
$\begin{array}{l}\text { Accuracy } \\
\text { Repeatability over the time } \\
\text { Long learning curve }\end{array}$ \\
$\begin{array}{l}\text { Lack of standardised definitions and scoring systems } \\
\text { for all findings }\end{array}$ \\
$\begin{array}{l}\text { Bedside procedure for rheumatologist } \\
\text { Utilization within the rheumatology clinic }\end{array}$ \\
$\begin{array}{l}\text { Optimal patient acceptance } \\
\text { Multiregional evaluation in the same scanning session } \\
\text { Short exam duration } \\
\text { Monitoring of disease progression } \\
\text { Follow-up of the response to treatment } \\
\text { US guided procedures }\end{array}$ \\
\hline
\end{tabular}

\section{Advantages and disadvantages}

Advantages and disadvantages of US in OA are reported in Table 3.

US evaluation of osteoarthritic joints has a large number of advantages over other imaging modalities, which are commonly applied in the assessment of rheumatic pathology. The most evident is characterised by the possibility of using it as many times as required and without contraindications during subsequent patient clinical assessments [3]. It is, in fact, considered a safe, accurate and noninvasive tool that represents a real bedside procedure, being widely applicable during routine clinical practice. It has no radiation hazard and no contraindications for patients, who readily accept and tolerate it without any difficulties, appreciating to be examined in the course of the rheumatologic evaluation with a US examination of short duration [2]. Sonographic equipment are widely available in most hospitals and outpatient units and this can facilitate the use of US in the rheumatologic clinical activity, thus avoiding requests for examinations to radiology units and thereby reducing waiting lists. US focusses a multiregional evaluation of the musculoskeletal system in the same scanning session and permits an extensive evaluation of most changes appearing in OA with the possibility of monitoring disease progression and performing a follow-up of the response to both local and systemic treatment $[3,5]$. Various procedures such as fluid aspirations within joint and periarticular tissues, local drug injections and biopsy are executed with safety, precision, reliability and optimal patient tolerance under sonographic guidance [37,60,61].

The most relevant disadvantage of US in OA consists of its partial accessibility to the inner joint structures, resulting in frequent difficulties in the complete visualisation of the hyaline cartilage in most joint sites [17]. This depends on the limited number and width of acoustic windows for sonographic beam penetration, due to its inaccessibility beyond the bony cortex [3,11]. Similarly to other imaging modalities, US is considered an operator-dependent technique, which, particularly for sonography, is related both to image acquisition and interpretation [3,5]. The recent technological improvement with development of new high-quality equipment has partially solved this particular problem, facilitating the visualisation of joint structures and the detection of their possible involvement. Moreover, the recent production of more sophisticated equipment has markedly increased their costs, thus considerably raising expenses related to the acquisition of the instruments [3]. On the contrary, US has a considerably low running cost, especially when compared with other imaging tools. It needs a sensibly long learning curve to obtain good experience, thus determining the necessity, on the one hand, of having considerable free time to acquire competency and, on the other hand, of being highly motivated operators. The lack of standardised definitions and scoring systems indicative of OA and the absence of solid evidence regarding the reliability of US in demonstrating the characteristic osteoarthritic joint changes represent other disadvantages for which expert groups are working, they 
will be hopefully overcome in the near future [11]. In this sense, encouraging progress has been recently carried out with the demonstration that US has a good reproducibility in articular cartilage thickness measurement by multiple examiners [17].

\section{Literature review}

Analysing the role of US as an assessment tool in OA, for the first time, a recent systematic review has shed light on published literature on this issue [12]. Two other recent systematic reviews had previously examined the validity of US as an outcome measure according to the basic components of the OMERACT filter, demonstrating that most of the work undertaken so far has considered inflammatory arthritis, and only a few manuscripts pertained to either synovitis or Doppler modalities in OA [13,14]. Keen and colleagues, after analysing all published literature which used sonography to assess joint changes in patients with $\mathrm{OA}$, underline that more work is needed to develop standardised definitions of pathology and to demonstrate the validity of sonography in OA [12]. Focussing in particular on the pathology imaged, the definitions used, scoring systems and their metric properties, results show that only a minority of works used US for assessing structural damage in OA [15,23-25]. Even fewer publications have analysed the role of Doppler and contrast agents [34,42-45]. Work performed until now demonstrates high variability not only in the pathology assessed but also in clear definition and grading of pathology. A lack of data on construct and criterion validity for US in OA is evident as well as deficiency of demonstrations on reliability and sensitivity to change [12].

\section{Practice points}

Sonography is commonly recognised as the reference imaging modality for assessing soft tissues abnormalities and, in recent years, its high sensitivity in showing bony cortex changes such as erosions has been widely reported. US has many indications in the assessment of patients with OA. They mostly include the possibility of demonstrating both structural damage and related joint inflammation even in early disease. Sonography has been shown to be a valuable tool in the detection of cartilage lesions, demonstrating not only early cartilaginous irregularities but also progressive thinning and deteriorations in late disease. Bony cortex involvement may be clearly depicted with the detection of osteophytes in most joints. In erosive hand OA, US may show the presence of erosions. In the case of synovitis, US images joint effusion, synovial thickening and proliferation and increased local vascularity, this last finding being related to active inflammation. Periarticular soft tissues involvement is also assessed with demonstration of local pathology, mainly represented by bursitis. The use of high-level equipment with multi-frequency, high-resolution transducers and high-sensitivity Doppler is mandatory for the accurate study of joint involvement in OA. Correct gray-scale and Doppler settings are fundamental requirements for optimising tissue visualisation in the finest details and for detecting their possible involvement (Table 2). US represents a valuable guidance of needles for joint and adjacent soft tissues aspirations, local drug injections and biopsy [37]. Thanks to all these characteristics, US can be used as a valuable bedside modality in the assessment of osteoarthritic patients, in monitoring disease progression and in the follow-up of response to therapy [3,11].

In the near future, development of more studies analysing different components of the OMERACT filter in OA will probably improve its role in research fields, demonstrating its improving value.

It is not yet clear whether new sonographic tools such as 3D-US, fusion imaging and elastography will add additional value to standard US [62]. Further studies are needed to deepen this aspect. However, very recent years have witnessed an increasing interest in research in this field, confirming a more widespread use of this tool for imaging different aspects of OA [15-19].

- US is the reference imaging tool for assessing soft tissue abnormalities

- US has high value in demonstration of inflammatory changes and structural damage in OA

- US shows joint changes related both to early and late disease in OA 
- The use of high-level equipment with high-resolution probes is mandatory for the accurate detection of changes in the minute details

- Correct gray-scale and Doppler settings have relevant value for imaging joint structures and their changes in minute particulars

- US is a bedside procedure that complements clinical evaluation of the osteoarthritic patient

- US enables to monitor disease progression and response to therapy from early to late disease

- Development of more studies analysing different components of the OMERACT filter in OA will improve its role in research field in the near future

- It is not yet clear whether new sonographic tools such as 3D-US, fusion imaging and elastography will give additional value to conventional US

\section{Research agenda}

In fact, research related to the applications of US for investigating different aspects of OA seems to concentrate mainly on correlations between synovitis and structural damage. This aspect has been recently analysed, particularly in hand and knee OA [21,34]. Comparisons with X-ray detected structural damage have underlined that US is a useful tool for research [18].

Recently, development of a preliminary US scoring system for hand OA provided interesting basis for the development of an US outcome tool that has the potential to be used in clinical trials [63].

Interesting research has been performed over the past few years with the attempt to overcome the lack of standardisation in US for definitions and scoring systems indicative of OA and, in particular, for hyaline cartilage evaluation. Encouraging progress has been recently carried out by the demonstration of a good reproducibility of US in measurement of knee articular cartilage thickness in cadaver specimens by multiple examiners [17].

The recent interesting development of promising research, which focusses on the definitions, detection and grading of elementary lesions, including scoring systems of inflammatory changes and structural damage, indicates the necessity of standardised measures for assessing osteoarthritic joints.

Technological novelties, which may represent valuable new applications in the near future and may add significant value to US assessment of joints in OA, are represented by the production of volumetric probes and intra-operating arthroscopic transducers and by the application of fusion imaging and 3D-US [1,3,62].

In particular, impressive expectations seem to be related to researches on fusion imaging. This new tool performs image superposition of US and either MRI or computed tomography (CT), producing realtime contemporary visualisation of sonographic and correlated MRI/CT images. This particular image overlapping procedure gives a wealth of information, offering a very complete set of data that could not be got by their serial use, thus enhancing information provided by either technique with accurate anatomical and functional correlations.

Another new and promising tool is represented by 3D-US, in which a volume of echoes is automatically acquired by the US system with a single placement of the transducer over the target area [62]. This interesting technique seems to improve problems related to image acquisition and standardisation of the US scanning technique. In fact, volumetric images provide infinite sets of data, depicting single anatomic structures and their involvement in OA in the finest details and rectifying problems related to the operator dependency on the acquisition process. This revolutionary US technique seems to show some of the most promising results in OA, particularly by its possibility to detect synovial proliferation and hypervascularity by using power Doppler $[62,64]$.

Further studies are needed to demonstrate the real value of these new modalities and to improve ultrasonographic approach to osteoarthritic joint.

- Development of research aimed to assess correlations between inflammation and structural damage in $\mathrm{OA}$

- Research focussing on standardised definitions, detection and grading of elementary lesions in OA

- Works aiming to demonstrate the validity of US in OA 
- Studies on scoring systems for inflammatory changes and structural damage in $\mathrm{OA}$

- Assessment of the role of US/MRI and US/CT fusion imaging in OA, including anatomical and functional correlations

- Analysis of the role of 3D-US for detection and monitoring of inflammation and structural damage in OA, including the application of Doppler modalities for follow-up of active inflammatory changes

\section{Conclusion}

Sonography has demonstrated to be a valuable imaging tool for assessing osteoarthritic joints. In early and late disease, it shows different changes resulting from inflammation and structural damage. They mainly consist of the appearance of joint effusion and synovial hypertrophy in presence of inflammation, and on the evidence of cartilage lesions and osteophytes in case of structural damage. The detection of Doppler signal within synovial tissue represents the mark of local inflammatory activity. Further but inconstant findings in different anatomic districts consist of bursitis, joint capsule thickening, meniscal protrusions, erosions and mucous cysts. US permits to locate and examine the extent of tissue involvement in single and multiple joints during the same scanning session, and to perform a wide and deep evaluation of the severity of different osteoarthritic lesions. Monitoring of disease progression and follow-up of the response to local and systemic treatments represent significant advantages of this imaging modality, which make it particularly appropriate for use in clinical practice. The execution of US guided procedures with safety and reliability has a relevant value in patient management.

In fact, unanswered questions are mainly represented by the future application of US on the basis of new technical and technological acquisitions. In particular, future improvements in US research on OA with the execution of studies investigating new aspects of the disease and using novel US tools such as 3D-US, fusion imaging and elastography will hopefully amplify the diagnostic abilities of sonography, analysing early and late disease with more accuracy and in minute detail.

\section{References}

*[1] Meenagh G, Filippucci E, Iagnocco A, et al. Ultrasound imaging for the rheumatologist VIII. Ultrasound imaging in osteoarthritis. Clin Exp Rheumatol 2007;25:172-5.

[2] Grassi W, Cervini C. Ultrasonography in rheumatology: an evolving technique. Ann Rheumentol Dis 1998;57:268-71.

[3] Filippucci E, Iagnocco A, Meenagh G, et al. Ultrasound imaging for the rheumatologist. Clin Exp Rheumatol 2006;24: $1-5$.

[4] Manger B, Kalden JR. Joint and connective tissue ultrasonography-a rheumatologic bedside procedure? A German experience. Arthritis Rheum 1995;38:736-42.

[5] Kane D, Grassi W, Sturrock R, et al. Musculoskeletal ultrasound - a state of the art review in rheumatology. Part2: clinical indications for musculoskeletal ultrasound in rheumatology. Rheumatology 2004;43:829-38.

[6] Meenagh G, Filippucci E, Kane D, et al. Ultrasonography in rheumatology: developing its potential in clinical practice and research. Rheumatology 2007;46:3-5.

[7] Grassi W, Filippucci E. Ultrasonography and the rheumatologist. Curr Opin Rheumatol 2007;19:55-60.

[8] Iagnocco A, Filippucci E, Meenagh G, et al. Ultrasound imaging for the rheumatologist. Ultrasonography of the shoulder. Clin Exp Rheumatol 2006;24:6-11.

*[9] Grassi W, Filippucci E, Farina A. Ultrasonography in osteoarthritis. Semin Arthritis Rheum 2005;34:19-23.

[10] Hunter DJ, Conaghan PG. Imaging outcomes and their role in determining outcomes in osteoarthritis and rheumatoid arthritis. Curr Opin Rheumatol 2006;18:157-62.

*[11] Möller I, Bong D, Naredo E, et al. Ultrasound in the study and monitoring of osteoarthritis. Osteoarthr Cartil 2008;16 [Epub 2008 Aug 2009].

*[12] Keen HI, Wakefield RJ, Conaghan PG. A systematic review of ultrasonography in osteoarthritis. Ann Rheumentol Dis 2009; 68:611-9.

[13] Joshua F, Edmonds J, Lassere M. Power Doppler Ultrasound in musculoskeletal disease: a systematic review. Semin Arthritis Rheum 2009;36:99-108.

[14] Joshua F, Lassere M, Bruyn G, et al. Summary findings of a systematic review of the ultrasound assessment of synovitis. J Rheumatol 2007;34:839-47.

*[15] Iagnocco A, Filippucci E, Ossandon A, et al. High-resolution ultrasonography in detection of bone erosions in patients with hand osteoarthritis. J Rheumatol 2005;32:2381-3.

*[16] Naredo E, Cabero F, Palop MJ, et al. Ultrasonographic findings in knee osteoarthritis: a comparative study with clinical and radiographic assessment. Osteoarthr Cartil 2005;13:568-74. 
*[17] Naredo E, Acebes C, Möller I, et al. Ultrasound validity in the measurement of knee cartilage thickness. Ann Rheumentol Dis 2009;68:1322-7.

*[18] Keen HI, Wakefield RJ, Grainger AJ, et al. Can ultrasonography improve on radiographic assessment in osteoarthritis of the hands? A comparison between detected pathology. Ann Rheumentol Dis 2008;67:1116-20.

[19] Lee CL, Huang MH, Chai CY, et al. The validity of in vivo ultrasonographic grading of osteoarthritic femoral condylar cartilage: a comparison with in vitro ultrasonographic and histologic gradings. Osteoarthr Cartil 2008;16:352-8.

[20] Dieppe P. Osteoarthritis and related disorders. Introduction and history. In: Klippel JH, Dieppe PA, editors. Rheumatology. London: Mosby; 1998. 8.1.1-8.1.2.

*[21] Keen HI, Wakefield RJ, Grainger AJ, et al. An ultrasonographic study of osteoarthritis of the hand: synovitis and its relationship to structural pathology and symptoms. Arthritis Rheum 2008;59:1756-63.

[22] Tarhan S, Unlu Z. Magnetic resonance imaging and ultrasonographic evaluation of the patients with knee osteoarthritis: a comparative study. Clin Rheumatol 2003;22:181-8.

[23] Aisen AM, McCune WJ, MacGuire A, et al. Sonographic evaluation of the cartilage of the knee. Radiology 1984;153: $781-4$.

[24] McCune WJ, Dedrick DK, Aisen AM, et al. Sonographic evaluation of osteoarthritic femoral condylar cartilage. Correlation with operative findings. Clin Orthop Relat Res 1990;254:230-5.

[25] Iagnocco A, Coari G, Zoppini A. Sonographic evaluation of femoral condylar cartilage in osteoarthritis and rheumatoid arthritis. Scand J Rheumatol 1992;21:201-3.

[26] Hattori K, Takakura Y, Ishimura M, et al. Differential acoustic properties of early cartilage lesions in living human knee and ankle joints. Arthritis Rheum 2005;10:3125-31.

[27] Castriota-Scanderbeg A, De Micheli V, Scarale MG, et al. Precision of sonographic measurement of articular cartilage: inter- and intraobserver analysis. Skeletal Radiol 1996;25:545-9.

[28] Qvistgaard E, Torp-Pedersen S, Christensen R, et al. Reproducibility and inter-reader agreement of a scoring system for ultrasound evaluation of hip osteoarthritis. Ann Rheumentol Dis 2006;65:1613-9.

[29] de Miguel Mendieta E, Cobo Ibáñez T, Usón Jaeger J, et al. Clinical and ultrasonographic findings related to knee pain in osteoarthritis. Osteoarthr Cartil 2006;14:540-4.

[30] Acebes JC, Sanchez-Pernuate O, Diaz-Oca A, et al. Ultrasonographic assessment of Baker's cysts after intra-articular corticosteroid injection in knee osteoarthritis. J Clin Ultrasound 2006;34:113-7.

[31] Benito MJ, Veale DJ, Fitzgerald O, et al. Synovial tissue inflammation in early and late osteoarthritis. Ann Rheumentol Dis 2005;64:1263-7.

[32] Blackburn Jr WD, Chivers S, Bernreuter W. Cartilage imaging in Osteoarthritis. Semin Arthritis Rheum 1996;25: 273-81.

[33] Conaghan P, D’Agostino MA, Ravaud P, et al. EULAR report on the use of ultrasonography in painful knee osteoarthritis. Part2: exploring decision rules for clinical utility. Ann Rheumentol Dis 2005;64:1710-4.

[34] Song IH, Althoff CE, Hermann KG, et al. Contrast-enhanced ultrasound in monitoring the efficacy of a bradykinin receptor 2 antagonist in painful knee osteoarthritis compared with MRI. Ann Rheumentol Dis 2009;68:75-83.

[35] Grassi W, Lamanna G, Farina A, et al. Sonographic imaging of normal and osteoarthritic cartilage. Semin Arthritis Rheum 1999;28:398-403.

[36] Grassi W, Filippucci E, Farina A, et al. Sonographic imaging of the distal phalanx. Semin Arthritis Rheum 2000;29:379-84.

[37] Epis O, Iagnocco A, Meenagh G, et al. Ultrasound for the rheumatologist. XVI. Ultrasound Guided Procedures 2008;26: 515-8.

[38] Qvistgaard E, Christensen R, Torp-Pedersen S, et al. Intra-articular treatment of hip osteoarthritis: a randomized trial of hyaluronic acid, corticosteroid, and isotonic saline. Osteoarthr Cartil 2006;14:163-70.

[39] Robinson P, Keenan AM, Conaghan PG. Clinical effectiveness and dose response of image-guided intra-articular corticosteroid injection for hip osteoarthritis. Rheumatology 2007;46:285-91.

[40] Filippucci E, Iagnocco A, Meenagh G, et al. Ultrasound for the rheumatologist. IV Ultrasonography of the hand and wrist. Clin Exp Rheumatol 2006;24:118-22.

[41] Iagnocco A, Epis O, Delle Sedie A, et al. Ultrasound for the rheumatologist. XVII. Role of colour Doppler and power Doppler. Clin Exp Rheumatol 2008;26:759-62.

[42] Walther M, Harms H, Krenn V, et al. Synovial tissue of the hip at power Doppler US: correlation between vascularity and power Doppler US signal. Radiology 2002;225:225-31.

[43] Walther M, Harms H, Krenn V, et al. Correlation of power Doppler sonography with vascularity of the synovial tissue of the knee joint in patients with osteoarthritis and rheumatoid arthritis. Arthritis Rheum 2001;44:331-8.

[44] Kristoffersen H, Torp-Pedersen S, Terslev L, et al. Indications of inflammation visualized by ultrasound in osteoarthritis of the knee. Acta Radiol 2006;47:281-6.

[45] Schmidt WA, Völker L, Zacher J, et al. Colour Doppler ultrasonography to detect pannus in knee joint synovitis. Clin Exp Rheumatol 2000;18:439-44.

[46] Backhaus M, Burmester GR, Gerber T, et al. Guidelines for muscoloskeletal ultrasound in rheumatology. Ann Rheumentol Dis 2001;60:641-9.

[47] Schmidt WA, Schmidt H, Schicke B, et al. Standard reference values for musculoskeletal ultrasonography. Ann Rheumentol Dis 2004;63:988-94.

[48] Meenagh G, Iagnocco A, Filippucci E, et al. Ultrasound for the rheumatologist. IV Ultrasonography of the knee. Clin Exp Rheumatol 2006;24:357-60.

[49] Saï A, Chérin E, Gaucher H, et al. Assessment of articular cartilage and subchondral bone: subtle and progressive changes in experimental osteoarthritits using $50 \mathrm{MHz}$ echography in vitro. J Bone Min Res 1997;12:1378-86.

[50] Chiang EH, Laing TJ, Meyer CR, et al. Ultrasonic characterization of in vitro osteoarthritic articular cartilage with validation by confocal microscopy. Ultrasound Med Biol 1997;23:205-13.

[51] Hodler J, Resnick D. Current status of imaging of articular cartilage. Skeletal Radiol 1996;25:703-9.

[52] Spriet M, Girard CA, Foster S, et al. Validation of a $40 \mathrm{MHz}$ B-scan ultrasound biomicroscope for the evaluation of osteoarthritis lesions in an animal model. Osteoarthr Cartil 2005;13:171-9. 
[53] Mathiesen O, Konradsen L, Torp-Pedersen S, et al. Ultrasonography and articular cartilage defects in the knee: an in vitro evaluation of the accuracy of cartilage thickness and defect size assessment. Knee Surg Sports Traumatol Arthrosc 2004; $12: 440-3$.

[54] Saarakkala S, Laasanen MS, Jurvelin JS, et al. Quantitative ultrasound imaging detects degenerative changes in articular cartilage surface and subchondral bone. Phys Med Bio 2006;51:5333-46.

[55] Töyräs J, Nieminen HJ, Laasanen MS, et al. Ultrasonic characterization of articular cartilage. Biorheology 2002;39:161-9.

[56] Nieminen HJ, Töyräs J, Rieppo J, et al. Real-time ultrasound analysis of articular cartilage degradation in vitro. Ultrasound Med Biol 2002;28:519-25.

[57] D'Agostino MA, Conaghan P, Le Bars M, et al. EULAR report on the use of ultrasonography in painful knee osteoarthritis. Part1: prevalence of inflammation in osteoarthritis. Ann Rheumentol Dis 2005;64:1703-9.

[58] Wakefield R, Balint PV, Szkudlarek M, et al. Musculoskeletal ultrasound including definitions for ultrasonographic pathology. J Rheumatol 2005;32:2485-7.

[59] Iagnocco A, Coari G. Usefulness of high resolution US in the evaluation of effusion in osteoarthritic first carpometacarpal joint. Scand J Rheumatol 2000;29:170-3.

[60] Pourbagher MA, Ozalay M, Pourbagher A. Accuracy and outcome of sonographically guided intra-articular sodium hyaluronate injections in patients with osteoarthritis of the hip. J Ultrasound Med 2005;24:1391-5.

[61] Mandl LA, Hotchkiss RN, Adler RS, et al. Can the carpometacarpal joint be injected accurately in the office setting? Implications for therapy. J Rheumatol 2006;33:1137-9.

[62] Filippucci E, Meenagh G, Epis O, et al. Ultrasound for the rheumatologist. XIII. New trends. Three-dimensional Ultrasonography 2008;26:1-4.

*[63] Keen HI, Lavie F, Wakefield RJ, et al. The development of a preliminary ultrasonographic scoring system for features of hand osteoarthritis. Ann Rheumentol Dis 2008;67:651-5.

[64] Ju JH, Kang KY, Kim IJ, et al. Three-dimensional ultrasonographic application for analyzing synovial hypertrophy of the knee in patients with osteoarthritis. J Ultrasound Med 2008;27:729-36. 